05

\title{
Формирование оптических вихрей с помощью голограмм с асимметричным профилем штриха
}

\author{
() В.П. Аксенов ${ }^{1}$, В.Ю. Венедиктов ${ }^{2,3,9}$, А.А. Севрюгин ${ }^{2}$, И.М. Турсунов ${ }^{2}$ \\ ${ }^{1}$ Институт оптики атмосферы Сибирского отделения РАН, \\ 634055 Томск, Россия \\ ${ }^{2}$ Санкт-Петербургский государственный электротехнический университет „ЛЭТИ“, \\ 197376 Санкт-Петербург, Россия \\ ${ }^{3}$ Санкт-Петербургский государственный университет, физический фракультет, \\ 198504 Петергоф, Санкт-Петербург, Россия \\ ฯ e-mail: vlad.venediktov@mail.ru
}

Поступила в редакцию 23.08.2017 г.

Представлены результаты экспериментального исследования возможности применения тонких голограмм с увеличенной за счет применения треугольного профиля штриха дифракционной эффективностью для формирования световых пучков с ненулевым орбитальным моментом (оптических вихрей). Показано, что в этом случае можно существенно (в теории - до 100\%) повысить эффективность преобразования без какого-либо искажения структуры формируемого пучка.

DOI: $10.21883 /$ OS.2018.02.45535.185-17

\section{Введение}

Световые пучки с оптическими вихрями (ОВ) [1-3] привлекают пристальное внимание исследователей и инженеров из-за их уникальных физических особенностей и многочисленных научно-технологических применений в таких, например, разных областях, как манипуляция микрообъектами, астрономия, оптическая связь. Оптический вихрь представляет собой волновую структуру с сингулярностью в поперечном распределении фазы (так называемой винтовой дислокацией волнового фронта) и нулевой амплитудой в центре ОВ. Он проявляет себя как изолированный минимум в поперечном распределении интенсивности светового пучка. Каждому такому минимуму может быть сопоставлен топологический заряд, равный $2 \pi m$ рад, где $m-$ целое число (положительное или отрицательное). Эта величина возникает как результат суммирования градиента фазы вдоль замкнутого контура, окружающего область с минимальной интенсивностью. Вектор Умова-Пойнтинга имеет в области оптического вихря циркулярную составляющую, приводящую к вихреподобному движению световой энергии. Следствием существования такой вихревой составляющей является наличие у лазерного пучка орбитального углового момента. Иногда сам волновой пучок, в структуру которого включен оптический вихрь, называют оптическим вихрем или вихревым пучком.

Оптические вихри возникают спонтанно: они сопровождают формирование спеклов при рассеянии лазерных пучков на шероховатой поверхности при распространении когерентного света в турбулентной атмосфеpe [4] или возникают во время лазерной генерации $[5,6]$. Их генерируют преднамеренно [7], получая вихревые пучки на выходе из лазерного резонатора, конвертируя фундаментальный гауссов пучок в вихревой пучок вне лазерного резонатора или применяя когерентную комбинацию пучков $[8,9]$. Во многих случаях конвертором служит фазовая голограмма [10-13].

В настоящей работе для генерации оптических вихрей также применяется голографический способ. Когерентный пучок линейно поляризованного монохроматического излучения $\mathrm{He}-\mathrm{Ne}$-лазера $(\lambda=0.63 \mu \mathrm{m})$ пропускается через маску-голограмму [12], которая синтезирована на компьютере. В соответствии с [12] в результате дифракции на ней в $\pm 1,2,3, \ldots$ порядках восстановятся оптические вихри с разным значением параметра $m$. Такие пучки по своей структуре близки к так называемым модам Лагерра-Гаусса с ненулевым орбитальным моментом [14], которые представляют немалый интерес для многих приложений. При этом сам профиль маски-голограммы, использованной авторами работы [12], является синусоидальным, и, как следствие, дифракционная эффективность такой голограммы невысока - не превосходит $30-40 \%$ в лучшем случае [15]. Чтобы повысить дифракционную эффективность голограммы, нами была синтезирована маскаголограмма с треугольным профилем, которая позволяет повысить энергетические значения $\pm 1,2,3, \ldots$ ит.д. порядков дифракции по сравнению с дифракцией на голограмме с синусоидальным профилем. Удобный алгоритм синтеза голограммы с треугольным профилем представлен в работах $[15,16]$, где предложен способ асимметризации профиля голографической решетки на основе сугубо локальной информации. Результаты исследования оптических вихрей, полученных голографическими методами на маске-голограмме с синусоидальным и треугольным профилями, представлены в настоящей работе. 

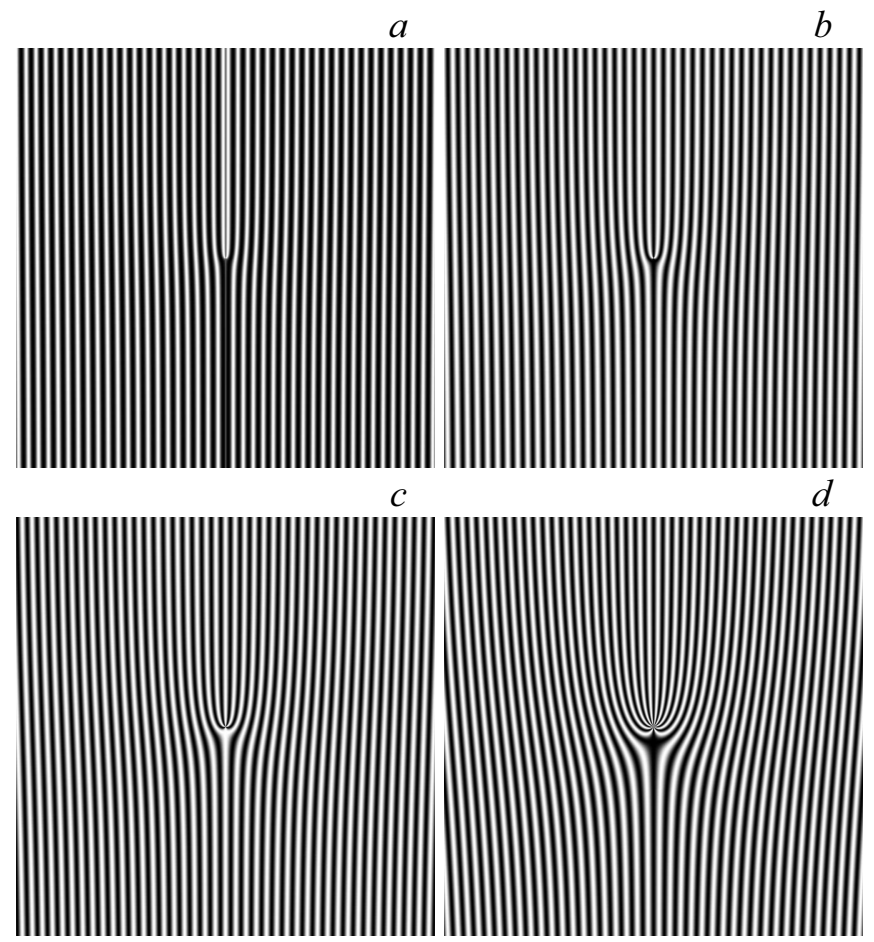

Рис. 1. Примеры масок-голограмм с различными значениями параметра $m: 1(a), 2(b), 4(c), 10(d)$.

\section{Синтез маски-голограммы}

Один из способов синтеза маски-голограммы цифровым методом состоит в построении интерференционной картины, интенсивность света в которой пропорциональна функции:

$$
H=\left|\psi_{1}+\psi_{2}\right|^{2}=\left|e^{i k x}+e^{i m \theta}\right|^{2}=2(1+\cos (k x-m \theta)),
$$

где $\psi_{1}=e^{i k x}, \psi_{2}=e^{i m \theta}-$ соответственно наклонная плоская волна и объектная волна; $(x, y)$ - декартовы координаты на плоскости; $\theta=\operatorname{arctg}\left(\frac{y}{x}\right)-$ полярная координата. Примеры синтезированных по соотношению (1) голограмм представлены на рис. 1.

Детально алгоритм синтеза маски выглядит следующим образом.

- Задать размеры исходного изображения по горизонтали $x_{0}$ и вертикали $y_{0}$ для каждого значения $i$ в пределах от 1 до $x_{0}$ включительно.

- Для каждого значения $j$ в пределах от 1 до $y_{0}$ включительно вычислить значение матрицы $H(x, y)=$ $=2\left(1+\cos \left(k x-m \cdot \operatorname{arctg}\left(\frac{y}{x}\right)\right)\right)$.

- Найти максимальный элемент $\max H=$ $=\max (H(x, y))$.

- Нормировать матрицу $H_{1}=H / \max H$.

- Получить матрицу по глубине цвета $H_{2}=255 H_{1}$.

- Привести матрицу к целым числам $H_{3}=\operatorname{int}\left(H_{2}\right)$.

- Построить изображение по матрице $H_{3}$.
Авторами были синтезированы маски-голограммы на языке программирования Python 3 в среде IDLE, которая предоставляется бесплатно и доступна для загрузки из сети Интернет.

\section{Асимметризация профиля маски-голограммы}

В работах $[15,16]$ предложен следующий цифровой способ асимметризации профиля голограммы, т. е. представление итоговой интерференционной картины с треугольным профилем.

Алгоритм построен в предположении, что интерферируют две когерентные волны равной интенсивности $I_{1}=I_{2}=I_{0}$. Результат интерференции этих волн описывается, как известно, функцией:

$$
I(x, y)=I_{0}(1+\cos [\Delta \varphi(x, y)]),
$$

где $\Delta \varphi(x, y)$ - разность фаз двух волн в данной точке плоскости с декартовыми координатами $x, y$.

Сама функция треугольного профиля может быть получена как

$$
F(x, y)=\frac{\operatorname{tg}\left(\frac{\Delta \varphi(x, y)}{2}\right)}{1+\left|\operatorname{tg}\left(\frac{\Delta \varphi(x, y)}{2}\right)\right|} .
$$

Функцию тангенса можно представить как

$$
\operatorname{tg}\left(\frac{\Delta \varphi(x, y)}{2}\right)=\frac{\sin [\Delta \varphi(x, y)]}{1-\cos [\Delta \varphi(x, y)]}
$$

График функции $F(x)=\frac{\operatorname{tg}(x)}{1+|\operatorname{tg}(x)|}$ представлен ниже на рис. 2.

Как видно из графика, эта функция отлично моделирует треугольный профиль, при этом дифракционная эффективность такого профиля при глубине фазовой модуляции $2 \pi$ будет близка к $100 \%$. Чтобы построить эту функцию, нужно обладать информацией о $\cos [\Delta \varphi(x, y)]$ и $\sin [\Delta \varphi(x, y)]$ в данной точке $(x, y)$. Картина с $\sin [\Delta \varphi(x, y)]$ может быть получена из $\cos [\Delta \varphi(x, y)]$ путем сдвига фазы на $\pi / 2$. Таким образом, достаточно обладать двумя голограммами, каждая из которых описывается функцией (2), фаза одной из которых смещена

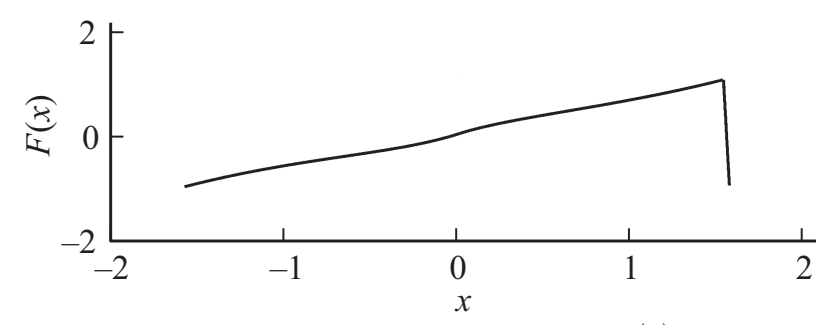

Рис. 2. График функции $F(x)=\frac{\operatorname{tg}(x)}{1+|\operatorname{tg}(x)|}$. 

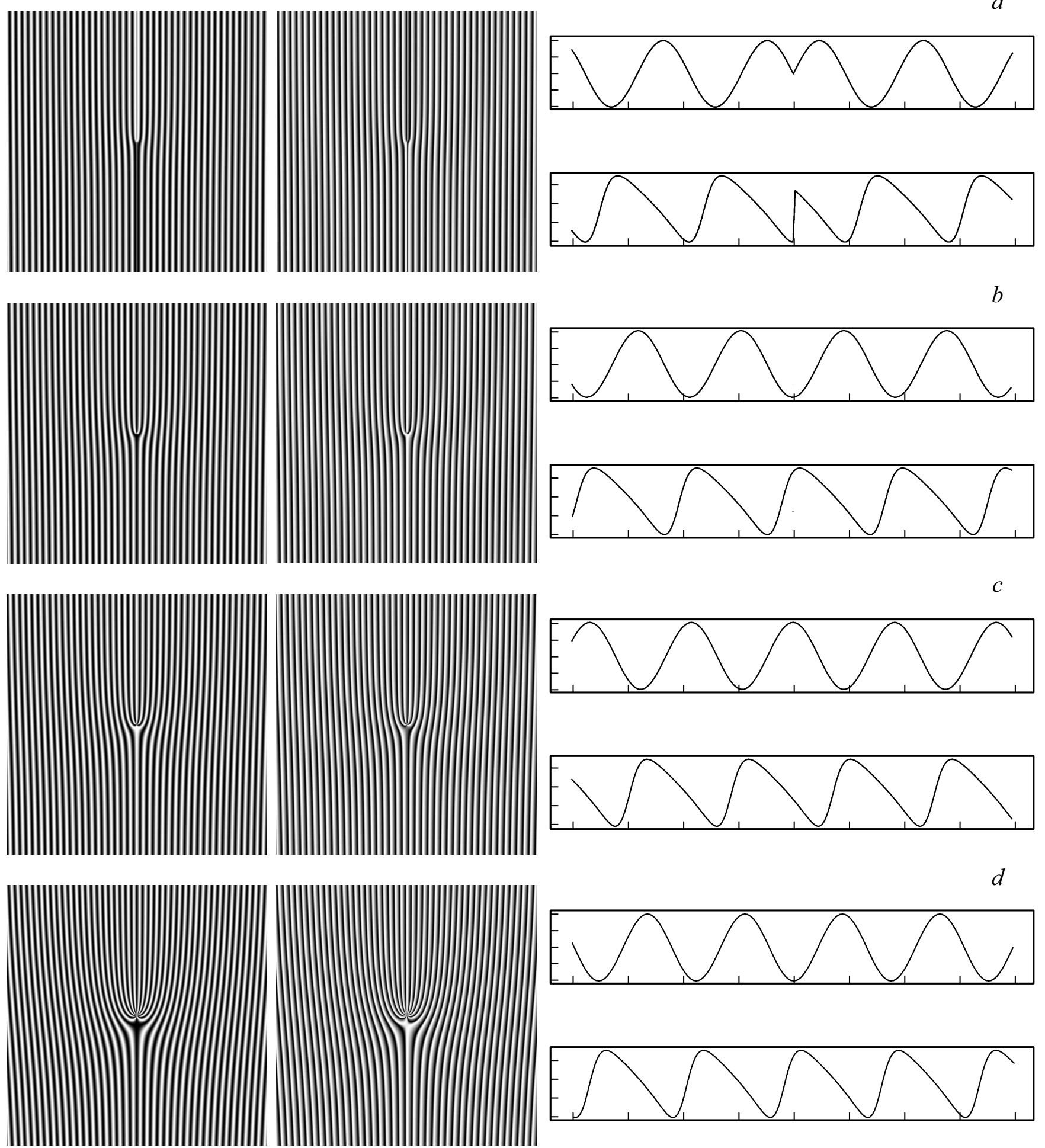

$d$
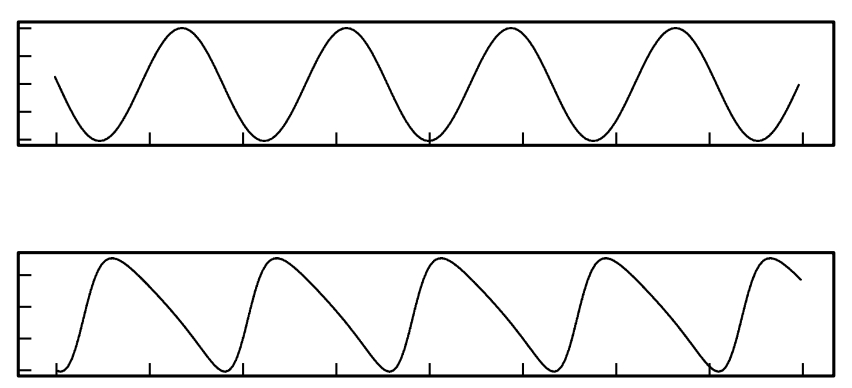

Рис. 3. Слева направо: маски-голограммы с синусоидальным и треугольным профилями и соответствующие им сечения плотности почернения (т. е. фазовой модуляции) вдоль оси $x$ при $y=0$ (для центральной области голограммы - 400 пикселей вдоль $x$ ). На рисунке представлены соответственно случаи $m=1(a), 2(b), 4(c), 10(d)$.

относительно другой на $\pi / 2$, чтобы построить голограмму с треугольным профилем. Достоинством метода является то, что для синтеза нужна лишь информация о текущей точке.
Итоговая интерференционная картина голограммы будет пропорциональна функции $F(x, y)$, а алгоритм построения маски-голограммы будет полностью аналогичен предыдущему алгоритму для случая синусоидаль- 
ного профиля, нужно лишь заменить в нем функцию $H(x, y)(1)$ на $F(x, y)(3)$ после подстановки в нее (4).

Примеры синтезированных голограмм с треугольным профилем и их сравнение с синусоидальным профилем вместе с графиками изменения интенсивности вдоль оси $x$ представлены на рис. 3 .

\section{Эксперименты с оптическими вихрями}

Для проверки изложенных соображений оптимальным вариантом было использование матричного пространственного модулятора света. В нашем распоряжении был модулятор компании Holoeye, модель LC-2002, на основе которого была создана экспериментальная установка, схема которой представлена на рис. 4.

Экспериментальная установка состоит из источника плоской монохроматической волны, образованного лазером 1 и коллиматором 2. Свет от источника разделялся светоделительным кубом 3 на две волны - опорную, которая направлялась на зеркало 4 , и объектную. Объектная волна падала на модулятор 5, на который с компьютера 6 подавалась маска-голограмма в виде видеосигнала, передаваемого по VGA-кабелю. Частота полос на маске-голограмме подбиралась исходя из достаточного расстояния между порядками дифракции при сохранении формы полос. Телескопическая система, образованная линзами 7 и 9 с фокусным расстоянием $1000 \mathrm{~mm}$ и диафрагмой в виде щели 8 , выделяла один из первых порядков дифракции, который и содержал необходимый оптический вихрь. Для обнаружения этого вихря использовался опорный пучок, который направлялся плоскими зеркалами 4,10 и кубом 11 под небольшим углом к объектному на матрицу цифровой камеры 12, где записывалась картина, образованная интерференцией между оптическим вихрем и опорным пучком с плоским фазовым фронтом. Для съемки дифракционных картин и оценки эффективности голографических масок камера 12 устанавливалась вместо диафрагмы 8 в фокальной плоскости линзы 7. Полученные результаты показаны на рис. 5.

Несмотря на сравнительно низкое разрешение модулятора, на полученных изображениях видно, что картина интерференции вихря и плоской волны не меняется в случае асимметризации маски-голограммы, в то время как дифракционная эффективность возрастает приблизительно на 20\%. Полученное значение прироста является

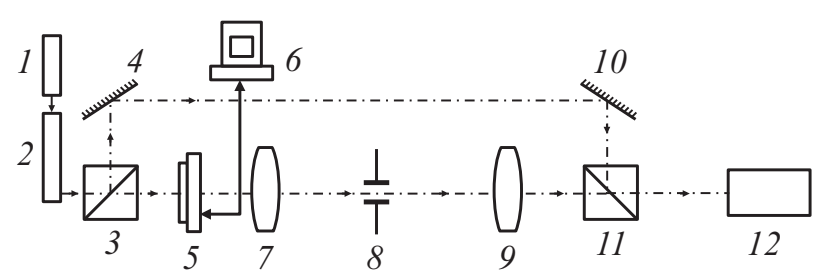

Рис. 4. Схема экспериментальной установки.
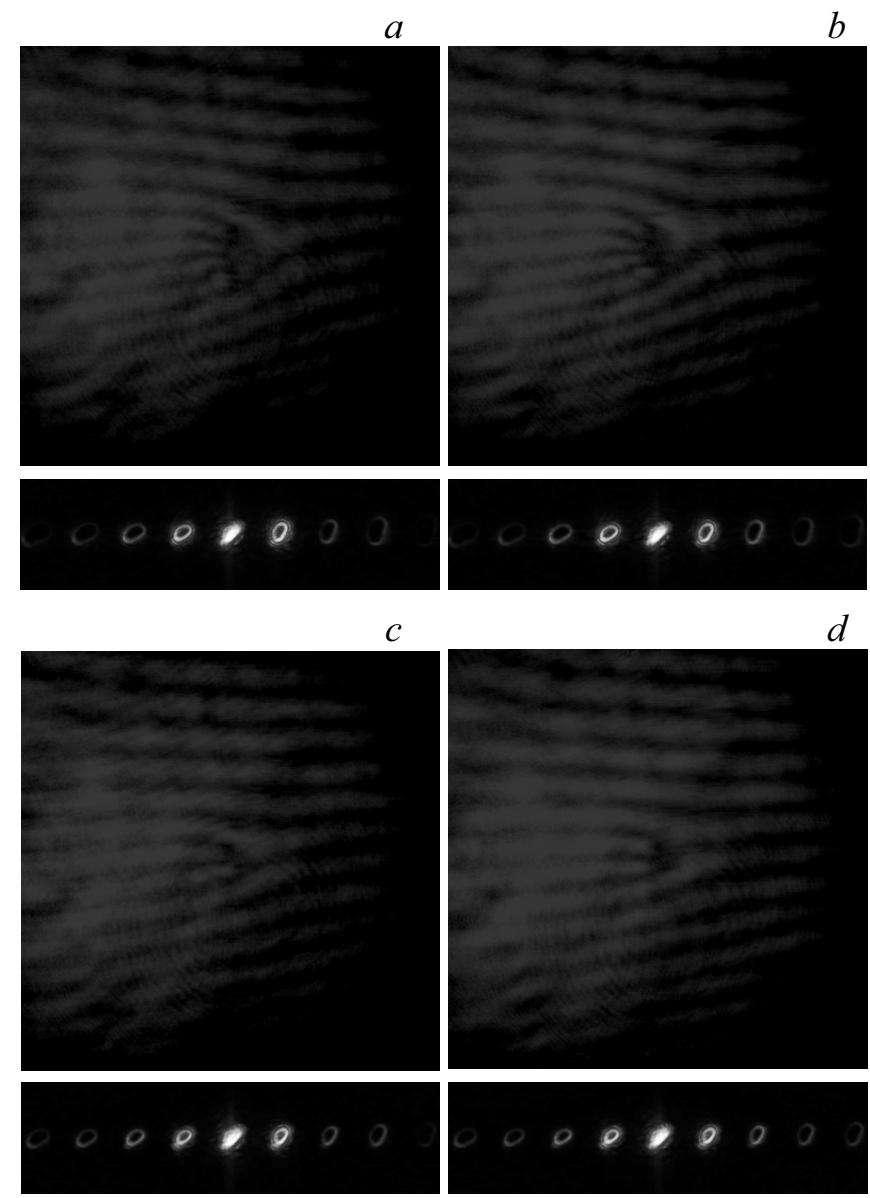

Рис. 5. Полученные результаты: верхняя часть - картина интерференции оптического вихря и плоского волнового фронта, нижняя часть - картина дифракции в фокальной плоскости линзы. (a) $m=4$, пилообразная решетка, $(b) m=4$, синусоидальная решетка, $(c) m=2$, пилообразная решетка, $(d)$ $m=2$, синусоидальная решетка.

приблизительным, рассчитанным на основании данных, полученных с КМОП матрицы камеры. Стоит отметить, что полученная нами дифракционная эффективность далека от идеальной ввиду невысокого разрешения матричного пространственного модулятора света, в результате чего профиль полос сильно квантован и заметно отличается от идеального.

\section{Заключение}

Нами успешно были синтезированы маски-голограммы с синусоидальным и треугольным профилями, благодаря которым возможно голографическим методом получать оптические вихри.

Полученные в результате эксперимента изображения показывают, что асимметризация профиля решетки не оказывает заметного влияния на получаемые картины интерференции оптического вихря и пучка с плоским волновым фронтом. Рост дифракционной эффективности 
в свою очередь составил около 20\%, несмотря на сильную ступенчатость профиля решетки из-за низкого разрешения пространственного модулятора света. При использовании более совершенных модуляторов света или масок высокого разрешения возможно еще большее увеличение дифракционной эффективности подобных масок.

В.Ю. Венедиктов и И.М. Турсунов признательны Минобрнауки РФ за финансирование в рамках Государственного задания № 8.1039.2017. В.Ю. Венедиктов также признателен СПбГУ за финансирование из средств Мероприятия № 6 поездки в Институт оптики атмосферы CO PAH, г. Томск. А.А. Севрюгин благодарит СПбГЭТУ „ЛЭТИ“ за поддержку в рамках проекта „Организация и проведение конкурса научноисследовательских проектов студентов, аспирантов и молодых научно-педагогических работников“.

\section{Список литературы}

[1] Allen L., Padgett M.J., Babiker M. // Prog. Opt. 1999. V. 39. P. 291-372.

[2] Bekshaev A.Ya., Soskin M.S., Vasnetsov M.V. Paraxial Light Beams with Angular Momentum. N.Y.: Nova Science Publishers, 2008.

[3] Dennis M.R., O'Holleran K., Padgett M.J. // Prog. Opt. 2009. V. 53 P. 293-363.

[4] Aksenov V.P., Tikhomirova O.V. // J. Opt. Soc. Am. A. 2001. V. 19. N 2. P. $345-355$.

[5] Arecchi F.T., Giacomelli G., Ramazza P.L., Residori S. // Phys. Rev. Lett. 1991. V. 67. P. 3749-3752.

[6] Harris M., Hill C.A., Tapster P.R., Vaughan J.M. // Phys. Rev. A. 1994. V. 49. P. 3119-3122.

[7] Yao A.M., Padgett M.J. Adv. // Opt. Photon. 2011. V. 3. P. 161-204.

[8] Розанов Н.Н. // Опт. и спектр. 1993. Т. 75. Вып. 4. C. 861-867.

[9] Аксенов В.П., Дудоров В.В., Колосов В.В. // Квант. электрон. 2016. Т. 46. № 8. Р. 726-732.

[10] Bazhenov V., Vasnetsov M.V., Soskin M.S. // JETP Lett. 1990. V. 52. P. 429-431.

[11] Heckenberg N.R., McDuff R., Smith C.P., White A. // Opt. Lett. 1992. V. 17. P. 221-223.

[12] Carpentier A.V., Michinel H., Salgueiro J.R. // Am. J. Phys. 2008. V. 76. N 10. P. 916-921.

[13] Mirhosseini M., Magaã-Loaiza O.S., Chen C., Rodenburg B., Malik M., Boyd R.W. // Opt. Express. 2013. V. 21. P. 30196-30203.

[14] Allen L., Barnett S.M., Padgett M.J. Optical angular momentum. Bristol: Institute of Physics Publ., 2003.

[15] Венедиктов В.Ю., Фрейганг Н.Н. // Опт. и спектр. 2008. T. 104. № 2. C. $338-343$.

[16] Венедиктов В.Ю., Ласкин В.А., Савинов В.А. // Опт. и спектр. 2010. Т. 109. № 4. С. 675-677. 